\title{
Safety Actuating Module for Transformer Relay Protection from Geomagnetically Induced Currents of HEMP
}

\author{
Vladimir Gurevich, Ph.D.* \\ Central Electrical Laboratory Israel Electric Corp., Haifa, Israel
}

*Corresponding Author: Vladimir Gurevich, Ph.D., Central Electrical Laboratory Israel Electric Corp., Haifa, Israel

\begin{abstract}
This article describes additional modules designed for co-use with a previously developed digital relay for transformer protection from geomagnetically-induced currents $(G I C)$, resulting from electromagnetic pulse of high-altitude nuclear explosion (HEMP). The safety actuating module (SAM) offered in the article prevents the transformer's tripping due to short-term GIC impacts or faulty actuation of digital relay as a result of any other reasons, for example at impact of electromagnetic interferences. The article discusses the SAM's hardware components, which ensure its maximum efficiency.
\end{abstract}

Keywords: HEMP, electromagnetic pulse, geomagnetically induced currents, GIC, power transformer

\section{INTRODUCTION}

Protection of power transformers of electrical grids from geomagnetically-induced currents (GIC) flowing in their earthed neutral conductors and resulting from E3 component of electromagnetic pulse of high-altitude nuclear explosion (HEMP), represents an important and up-to-date problem detailed and described in $[1,2]$.

A detailed description of a protection relay is presented in [3], developed by the author which protects transformers from GIC and a portable tester for periodic checks of the relay's serviceability. This relay is designed for automatic short-term tripping of a power transformer as a result of a direct current occurring in its neutral conductor, featuring high reliability and noise-resistance.

Actually, this kit fully solves the technical issue of the power transformers' protection in the power industry. Nevertheless, there is a problem, which is not connected with the equipment and significantly hampers the broad use of such a relay in electric grids. This is a psychological problem of power plants' and substations' personnel. They are afraid of faulty actuation of the relay as a result of any interference and tripping of the high-power transformer to which multiple high-voltage power lines are connected. Tripping of a powerful transformer with the earthed neutral conductor at a substation, and particularly a generator-transformer unit (the neutral conductors of which are always earthed) at a power plant, represents an emergency, which requires investigation and preparation of corresponding reports. This fear is reasonable. The offered relay should directly act upon the transformer's high-voltage circuit breaker (CB). At the same time, it is not standard equipment located in the open air outside the enclosed facility of the substation. It is directly connected to the substation's equipment through a long control cable running over the substation's (power plant's) territory. Actually, the designed relay [2] is well protected from faulty actuation resulting from both external electromagnetic fields and pulse interferences travelling along the control cables, but there is always room for doubt, especially when it comes to a non-standard protection relay.

\section{COMMISSIONING}

Considering the above-mentioned problem, the article offers: (1) step-by-step commissioning of the offered relay; and (2) use of special safety actuating module (SAM) designed to prevent faulty actuation of protection relay operating under the influence of environmental factors and powerful electromagnetic interference.

During the first stage (up to one year), the output contacts of the relay are expected to be connected into the alarm circuit rather than the tripping circuit of the transformer. It is suggested to use the simplest auxiliary module (Fig. 1) mounted in an electric cabinet or on a panel at a substation (or a power plant). 


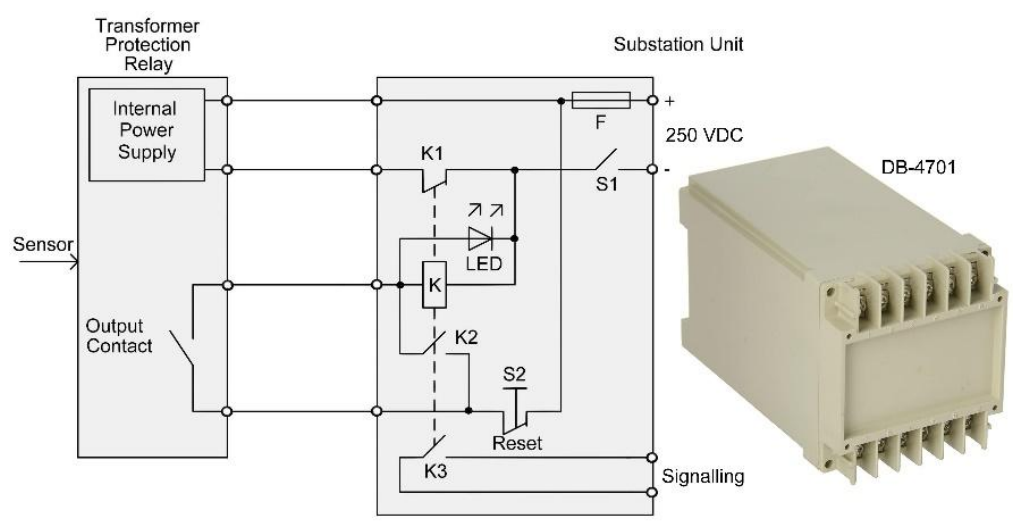

Figure1. The simplest auxiliary module designed to connect the transformer's protection relay during the first stage of commissioning.

The purpose of this stage is to prove high noise-resistance (no faulty actuation during the whole probation period) and serviceability (actuation upon periodic simulation of emergency mode by means of simulating tester) of the protection relay.

Upon successful completion of the first stage of testing, we can proceed to the second stage lasting several months, i.e. commissioning of SAM at a substation (power plant) and testing of its reliability.

\section{Safety ACtuating Module}

The module SAM developed by the author provides automatic blocking of the first actuation of protection relay, several second time-delay, rebooting of relay and waiting for its second actuation, if a signal from a sensor, which caused the first actuation to still be present after time-delay and rebooting. The SAM will switch off the transformer only upon completion of all these stages.

Obviously, when we deal with a very powerful electromagnetic interference such as HEMP, entering the equipment both as an electric field surge and as a high-voltage pulse arriving at input and output terminals along the power and control cables, the SAM must be built using electromechanical components (Fig. 2) rather than electronic microchips and microprocessors used in the modern controllers.

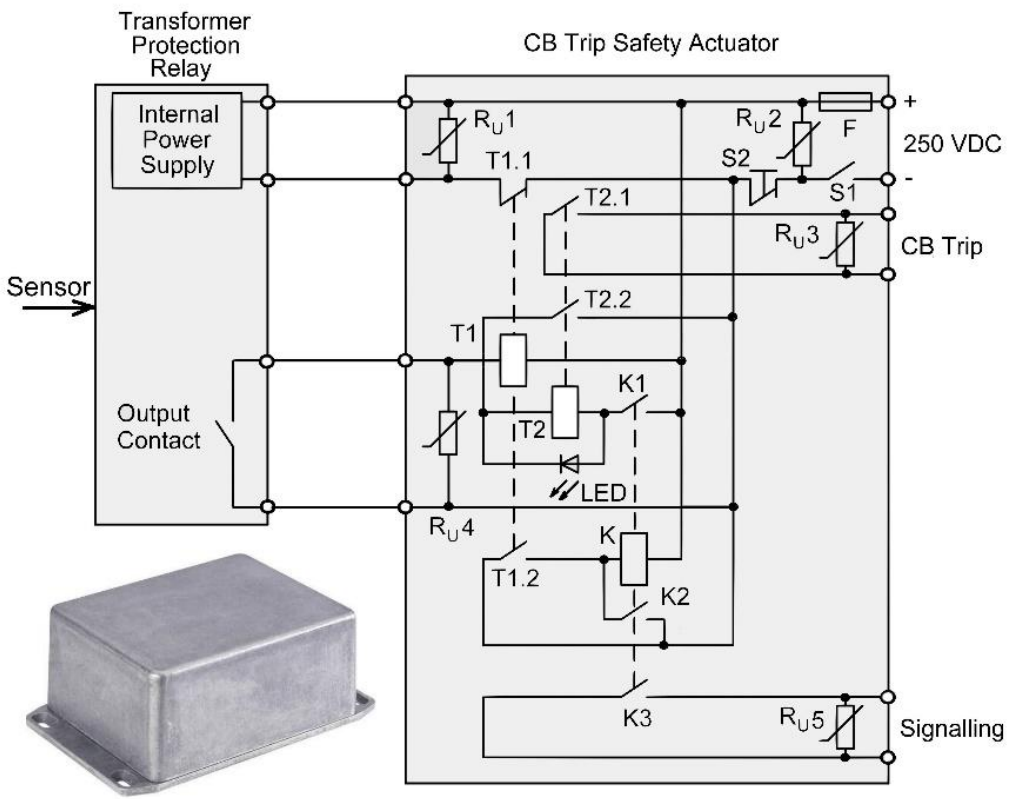

Figure2. Circuit diagram of SAM built using electromechanical components. T1 and T2 - timers with on-time delay

The operating principle of the SAM developed by the author is described below. During the waiting period (no signal from the sensor; the output contact of protection relay is open) T1 and T2 timers as 
well as $\mathrm{K}$ relay are deenergized. The T2.1 terminal (tripping of the transformer $\mathrm{CB}$ ) and $\mathrm{K} 3$ contact (alarm) are open.

In the case of relay actuation for whatever reason and closing of its output contact, the voltage from a 250 VDC substation auxiliary power system will be energized a T1 timer. The timer will start countdown. Four - five seconds later its T1.1 contact will open, while the T1.2 contact will close. Relay $\mathrm{K}$ will switch on and power itself via its own $\mathrm{K} 2$ contact simultaneously delivering power to the $\mathrm{T} 2$ timer via K1 contact. Timer T2 will start, but immediately stop the countdown upon the break of the relay's supply circuit by the T1.1 terminal and opening the relay protection output contact. The circuit will return to its initial status except for $\mathrm{K}$ relay (which will remain on) and its $\mathrm{K} 1, \mathrm{~K} 2$ and $\mathrm{K} 3$ contact (which will stay closed). A signal will be delivered to the alarm circuit.

If upon short-term outage and restoration of the protective relay's power its output contact is still open, the status of the SAM will remain the same. The S2 "Reset" button will break the alarm circuit and return the device to its initial state.

If actuation of the transformer relay protection was correct (based on a signal arriving from the sensor) repeated actuation of the relay and closing of its output contact will take place immediately upon restoration of the relay power supply through the T1.1 contact. The T1 timer will start countdown again, but now the T2 timer will energize and start countdown simultaneously via the $\mathrm{K} 1$ contact, which is still closed. The T2 timer's time delay is several times smaller than that of the T1 timer. As a consequence, it is the first to actuate generating the command to trip the transformer $\mathrm{CB}$ and enters the self-power mode. Four-five seconds later, the T1 timer will actuate again and open the power supply circuit of the protection relay through the T1.1 contact, but the power transformer will be off already by this time and the sensor signal will disappear. The S2 "Reset" button will bring the SAM into its initial state.

The SAM's varistors $\mathrm{R}_{\mathrm{U}} 1-\mathrm{R}_{\mathrm{U}} 5$ prevent high-voltage pulses from entering its input and output terminals, whereas the Faraday cage (i.e. aluminum case) ensures protection from the electromagnetic field.

\section{TIMERS FOR SAM}

The issue of choosing the correct timers for SAM is still open. This problem proved to be quite difficult as it initially acknowledged that common electronic timers on microprocessors and microchips are unacceptable. Are there non-electronic timers? For this answer we need to conduct a profound analysis on non-electronic timers.

The simplest and the most reliable type of non-electronic timers is represented by the electromagnetic timers' group. Their actuation delay is controlled by a thick-wall copper cylinder (i.e. a short-circuited coil) placed onto the core of the electromechanical relay (Fig. 3).

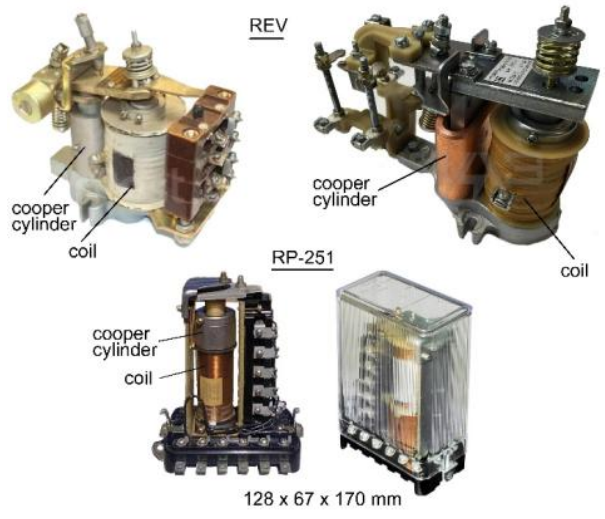

Figure3. Electromagnetic timers

Unfortunately, timers of this group provide maximum $0.1 \mathrm{sec}$ delay, only in addition to their bulky size and heavy weight.

Another type of non-electronic timer is represented by electropneumatic timers. Their actuation delay (or opening) is controlled by a solenoid and bellows (usually rubber) or a piston and a nipple with a hole to exhaust the air out or take it in to a chamber (Fig. 4).

International Journal of Research Studies in Electrical and Electronics Engineering (IJRSEEE) Page | 3 

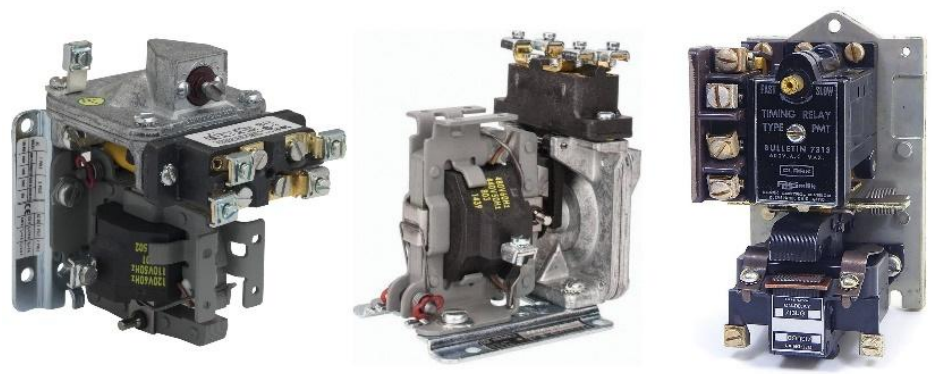

Figure4. Electropneumatic timers

Timers of this type are acceptable in terms of their time delay, but their size and weight...

The next type of studied non-electronic timer is represented by clock and spring-operated timers (Fig. 5).

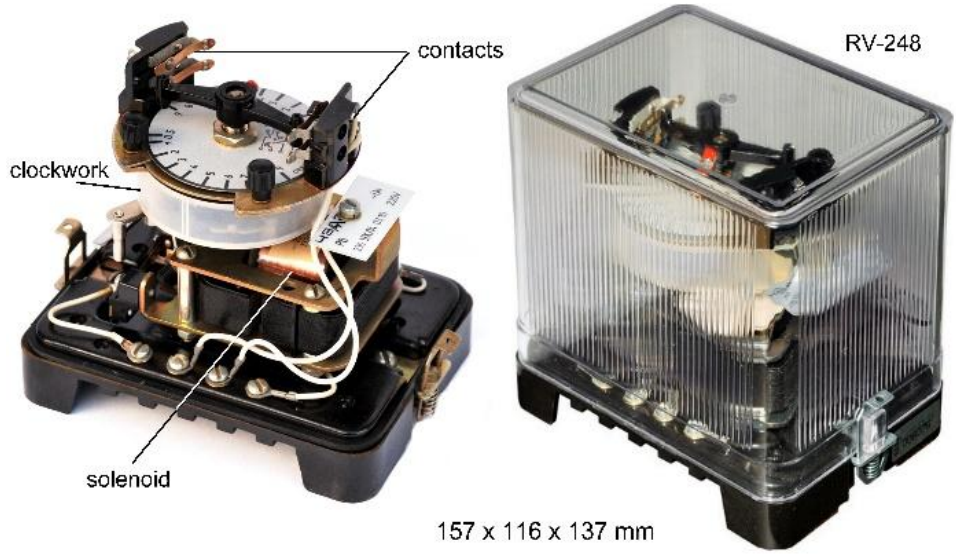

Figure5. Clock-operated timers

When power comes to a solenoid, it overcomes the tension of a pullback spring and enables the clock mechanism. As the time passes, the output spindle of the clock mechanism rotates and when it reaches the preset target value it will close the output contact and stop. These timers are quite reliable, accurate and provide the required time delays for the SAM. The only disadvantage of such a relay is their size.

Another type of studied non-electronic timer is represented by motor-driving timers (Fig. 6).

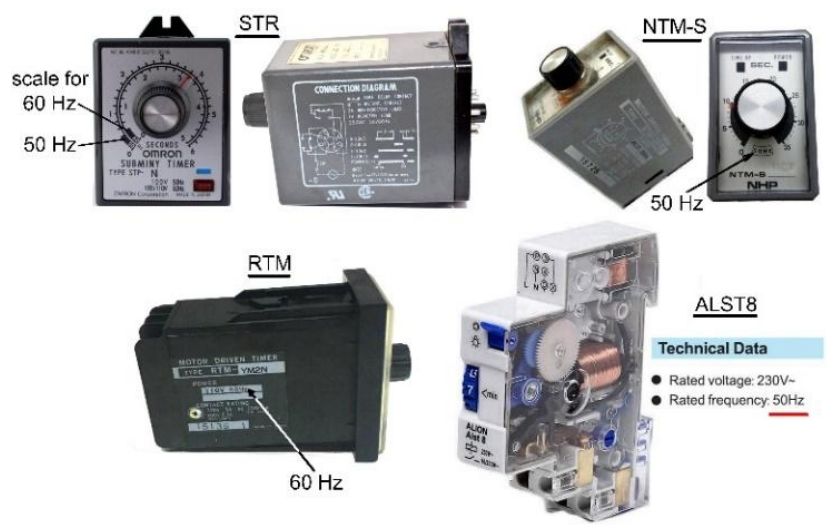

Figure6. Motor-driving timers built on miniature synchronous motors.

In these timers a small synchronous motor rotates a gear reducer shaft at the preset speed (determined by supply voltage frequency) and provides a required time delay after completion of preset rotations of the shaft. These timers are quite portable, reliable and provide time intervals required for the SAM. The drawback of these timers is a necessity to supply alternating voltage only with $50 \mathrm{or} 60 \mathrm{~Hz}$ frequency, which makes them unacceptable for the SAM. In fact, there is a limited number of motor-driven timers on the market that can be powered from a DC source, e.g. M6446 type (Fig. 7). 


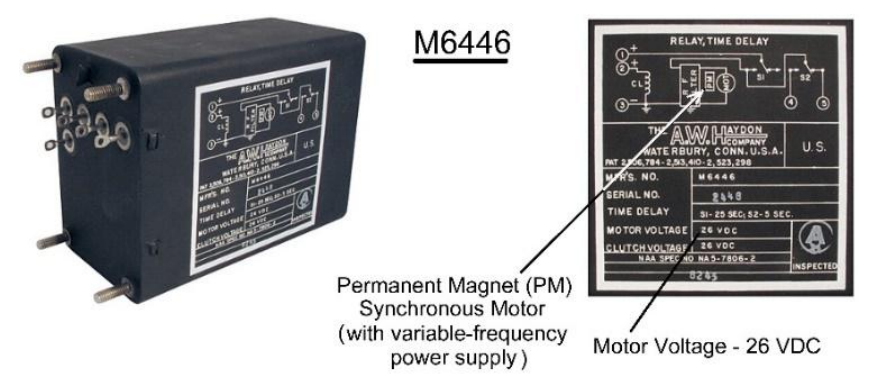

Figure7. DC motor-driven timer for 26V operating voltage

Timers of this type use synchronous motors with permanent magnets (PM-motors); while the required power frequency of the motor is provided by a built-in frequency converter. We cannot say that this is a non-electronic timer; and in addition, they are built for low supply voltage only (up to 28V).

\section{ESPECIALLY DESIGNED TIMER FOR SAM}

The above-mentioned analysis allows us to conclude that none of the available non-electronic timers are suitable for SAM for various reasons. That is why we developed a timer especially designed to be used in the SAM (Fig. 8).

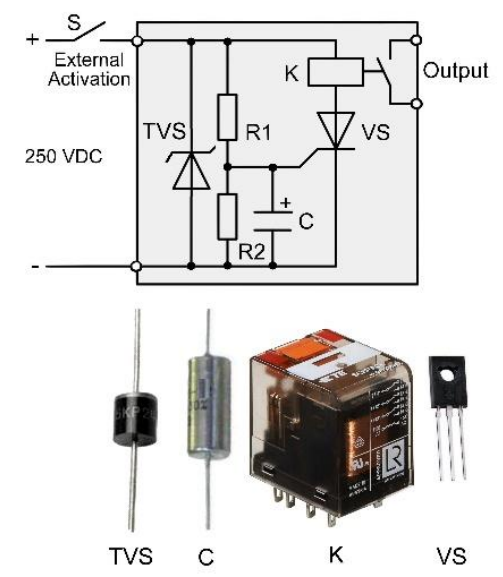

Figure8. Timer specially designed for the SAM.

This is not a $100 \%$ "non-electronic" timer, but it contains only one active electronic component, i.e. thyristor for $600 \mathrm{~V}$ operating voltage protected from surge overvoltages by 30KPA300A TVS-diode, with clamping voltage not exceeding 484V. Perfect specifications of this timer (simplicity, reliability, noise-resistance, parameter consistency, small size) and time delay intervals, ranging from fractions of a second to 10 seconds, are provided by an aggregate of elements with special characteristics. For example, thyristor VS (C106M) of the selected type combines sufficient capacity, high operating voltage and very small gate current (fractions of milliamp). This small current value makes it possible to use an R1 time-setting resistor (with resistance value of hundreds of thousands of Ohm) and an R2 resistor (with resistance value of several kilo-ohm) with a voltage dip of less than $1 \mathrm{~V}$ at 240VDC supply voltage. This, in turn, makes it possible to use a very stable hermetic tantalum 1000 microfarad capacitor T140D108K006A type in the timer (there are no capacitors of such a type featuring higher capacity) with $6.3 \mathrm{~V}$ operating voltage. The shelf-life of such capacitors is 25 years and more. This is very important for SAM timers as they can remain powerless for many years and need to ensure normal operability of SAMs after a long idle-time. Another special element of this circuit is the electromagnetic relay K, type PT370220 (Schrack) with winding resistance of 65 kilo-ohm. This is a unique winding resistance level for a miniature relay, causing a very low operating current of the relay (3.4 mA). This means that even a small thyristor (e.g. C106M type) operates with a good margin in terms of dissipated power and does not require any additional heat sink even when it is on for a long time. The circuit is protected from pulse interference due to a powerful TVS-diode mounted at the input and a large 
capacitor in the circuit of the thyristor "gate" electrode, which prevents it from opening upon a pulse interference impact. Moreover, it should be noted that with this layout of the timer and its connection to the SAM, it is maintained in a fully powerless state during the whole waiting period and thus it cannot result in faulty SAM actuation by itself.

\section{CONCLUSION}

The SAM described in the article prevents tripping of power transformers under a short-term GIC impact (which does not pose a threat for a transformer) or under faulty actuation of digital protection relay caused by whatever reason. The kit of devices developed by the author, which includes an electronic relay with a GIC sensor, simulating tester, additional module at a substation and a safety actuating module, solves the problem of HEMP protection of power transformers used in electric grids.

\section{REFERENCES}

[1] Gurevich V. Protection of Substation Critical Equipment Against Intentional Electromagnetic Threats. Wiley, 2017. $-228 \mathrm{p}$.

[2] Gurevich V. Protecting Electrical Equipment: Good Practices for Preventing High Altitude Electromagnetic Pulse Impacts. - De Gruyter, Berlin, 2019. - 386 p.

[3] Gurevich V. Protection of a Power Transformer from High Altitude Electromagnetic Pulse. - International Journal of Research Studies in Electrical and Electronics Engineering (IJRSEEE), Vol. 6, Iss. 1, 2020, pp. $17-24$.

Citation: Vladimir Gurevich, (2020) "Safety Actuating Module for Transformer Relay Protection from Geomagnetically Induced Currents of HEMP", International Journal of Research Studies in Electrical and Electronics Engineering (IJRSEEE), pp. 1-6, DOI: http://dx.doi. org/10.20431/2454-9436.0602001

Copyright: () 2020 Authors. This is an open-access article distributed under the terms of the Creative Commons Attribution License, which permits unrestricted use, distribution, and reproduction in any medium, provided the original author and source are credited. 